\title{
Recht auf Vergessen? Ethik der zweiten Chance?
}

\author{
Abbt, Christine
}

DOI: https://doi.org/10.1515/dzph-2016-0068

Other titles: Überlegungen zum Urteil des Europäischen Gerichtshofes (EuGH) vom 13.5.2014

Posted at the Zurich Open Repository and Archive, University of Zurich ZORA URL: https://doi.org/10.5167/uzh-133615

Journal Article

Published Version

Originally published at:

Abbt, Christine (2016). Recht auf Vergessen? Ethik der zweiten Chance? Deutsche Zeitschrift für Philosophie, 64(6):925-946.

DOI: https://doi.org/10.1515/dzph-2016-0068 


\title{
Christine Abbt* Recht auf Vergessen? Ethik der zweiten Chance?
}

\section{Überlegungen zum Urteil des Europäischen Gerichtshofes (EuGH) vom 13.5.2014}

DOI 10.1515/dzph-2016-0068

\begin{abstract}
The notion of 'forgetting' has assumed a new dimension in the digital age. Here I will examine a particular kind of forgetting as reflected in a ruling of the European Court of Justice (ECJ). What the ruling of the ECJ of May 13, 2014 (C-131/12) formulates and invokes as a "right to be forgotten" encompasses the right to co-determine whether certain personal data in the Web should immediately show up or not when a first name and surname is entered as part of a search. When a user has invoked the "right to be forgotten", and it is determined that it applies, information is, however, not made irretrievable. It continues to remain possible to find this information in a roundabout way, i.e., by means of more precise search queries, although the information should not immediately become visible the moment a person's full name is typed into a search engine. I will argue that this ruling can be seen as corroborating the fundamental rights of the individual. The idea of the "right to be forgotten" is to give a person a second chance in society. Not all forms of forgetting and remembering can be subsumed under this idea. As will be expounded, this court decision offers a useful normative fundament for the distinction between (1) legitimate attempts at reintegration, (2) legitimate attempts at rehabilitation and (3) unjustified recourse to a right to be forgotten.
\end{abstract}

Keywords: forgetting, right to be forgotten, personal rights, statute of limitations

*Kontakt: Christine Abbt, Universität Luzern, Philosophisches Seminar, Frohburgstraße 3, 6002 Luzern, Schweiz; christine.abbt@unilu.ch 


\section{1 „Vergessen“ im Kontext der Rechtsprechung des EuGH}

In Bezug auf eine philosophische Untersuchung zum Vergessen ist interessant, dass im juristischen Kontext überhaupt so übereinstimmend davon die Rede ist. Durch diesen Gebrauch werden jene Aspekte von Vergessen ins Zentrum gerückt, die willkürlich zu beeinflussen sind. Wenn es ein Recht auf Vergessen geben kann, was u. a. durch das im Nachfolgenden noch genauer zu betrachtende Urteil des EuGH nahegelegt wird, dann bedeutet dies zumindest, dass ein Konsens darüber besteht, dass bestimmte Formen von Vergessen eingefordert und durchgesetzt werden können. Vergessen wird dabei als ein Vorgang vorgestellt, der aktiv angestrebt und in Gang zu bringen ist. Dies ist umso bemerkenswerter, wenn man bedenkt, dass es hier um ein Vergessen von Daten geht, von denen manche Technologie-Spezialisten sagen, dass sie nie wieder vollständig eliminiert werden können, wenn sie erst einmal ins Netz eingespeist sind. ${ }^{1}$ Anfangs des 21. Jahrhunderts setzt sich damit offensichtlich eine Verwendung des Begriffs „Vergessen“ durch, der in den letzten Jahrzehnten des 20. Jahrhunderts wenig im allgemeinen Gebrauch war. Allerdings, und dies ist bezüglich des Sprachgebrauchs nicht unwichtig, ist der Ausdruck „Vergessen“ innerhalb des Urteiltexts des EuGH dort, wo nicht auf Vorlagen und Anfragen anderer Gerichte und deren Dokumente rekurriert wird, in Anführungszeichen gesetzt. ${ }^{2}$ Der EuGH bemüht sich darum, so macht es den Anschein, aufzuzeigen, dass der Begriff „Vergessen“ innerhalb des Urteils einerseits bewusst verwendet wird, dass die konkrete Verwendung jedoch andererseits dem gängigen Sprachgebrauch zuwiderläuft. „Vergessen“ bedeutet im hier diskutierten Urteil nämlich nicht Löschung, sondern Erschwerung des Zugriffs auf Daten.

Was als ein „Recht, vergessen zu werden“ formuliert und eingefordert wird, umfasst das Recht darauf, dass bestimmte Informationen im Netz über eine Person bei einer Suchanfrage nicht (sofort) erscheinen. Die Daten sind dadurch

\footnotetext{
1 Beate Wöhe vom Internet-Forum Channelpartner antwortet auf die immer wieder gestellte Frage danach, ob man eigene Spuren im Internet löschen könne: „Leider ist es unmöglich, alle Spuren aus dem Netz zu tilgen. Sie können Einträge löschen, aber tausende von Bots, Crawlern und Suchmaschinen sind jede Sekunde im Netz nach neuen, aber auch alten Informationen auf der Suche. Der beste Weg, sich aus dem Internet zu löschen ist leider der, dort niemals Informationen über sich hinterlassen zu haben“ (Wöhe 2015). Zum Problem, dass einmal hochgeladene Daten nicht mehr vollständig zu beseitigen sind und den ethischen Forderungen, die sich daraus ergeben, vgl. u. a. Zimmer (2010) und Tavani (2007).
}

2 Vgl. EuGH-Urteil, Randziffer 89. 
nicht unauffindbar. Sie müssen aber auf Umwegen, durch genauere Recherchefragestellungen, gefunden werden und springen nicht sofort ins Auge, wenn eine Suchmaschine mit der Eingabe eines Namens in Gang gesetzt wird. ${ }^{3}$ Suchmaschinenanbieter sind durch das Urteil des EuGH nicht dazu aufgefordert, bestimmte Daten zu löschen, sondern verpflichtet, sie unter Umständen bei einer Namenssuche nicht mehr zu zeigen. Aus verschiedenen Gründen - sowohl aus technischen (es ist kaum möglich, Daten, die einmal im Netz waren, vollständig zu eliminieren) als auch aus politischen (das Urteil des EuGH wird in den EU-Staaten umgesetzt und von den Suchmaschinenanbietern auch in den EWR- und EFTAStaaten ernst genommen, jedoch von den USA und vielen weiteren Ländern kritisiert und abgelehnt ${ }^{4}$ ) - wäre es ohnehin nicht durchsetzbar, dass Daten, die vorhanden sind, unauffindbar gemacht werden. Das „Recht, vergessen zu werden“ bedeutet hingegen auch nicht, dass Daten tatsächlich eliminiert werden sollen oder verloren gehen müssen. Es verhilft einzig dazu, dass Daten über eine Person gegebenenfalls nicht sofort in Kombination mit deren Namen auftauchen.

Ob sich durch ein solches Recht auf Vergessen die Bürden der omnipotenten Datenspeicherung mildern lassen, ist fraglich. An dem konkreten Fall, der für das Urteil des EuGH Anlass war, zeigen sich allzu deutlich die Verstrickungen und Wechselwirkungen zwischen „Vergessen“ und „Erinnern“. Jener Mann, der zuerst vor ein spanisches Gericht ging, weil er einen Suchmaschinenanbieter dazu bewegen wollte, einen Eintrag über ihn zu löschen, hat viel erreicht - allerdings nicht das Ziel, dass nicht alle sofort über ihn erfahren, dass sein Haus einmal zwangsversteigert werden musste. Heute wissen Unzählige nichts anderes von ihm als genau dies. ${ }^{5}$ Natürlich ist diese Sichtweise nur beschränkt zulässig, um über das Problem der Privatsphäre im Netz zu debattieren. Dennoch lässt sich in dieser Zuspitzung deutlich machen, was das Problem ist. Die Suchmaschinenanbieter, die nun unter Umständen angehalten sind, in europäischen Ländern Links

3 Die Erschwerung ist dabei doch recht eingeschränkt. Sobald neben dem Vornamen und Nachnamen noch ein geeignetes weiteres Stichwort in das Suchfeld eingegeben wird, erscheint der Link mit den allenfalls unerwünschten Daten immer noch.

4 Vgl. dazu etwa Weber/Heinrich (2014). Die Autoren diskutieren dabei insbesondere auch die Frage, ob es um die Schaffung eines neuen Grundrechts auf Vergessen(werden) gehe(n könne) und wie dieses gegebenenfalls mit der Meinungsäußerungsfreiheit vereinbar sein bzw. in Einklang gebracht werden könnte. Zu dieser Frage hat sich der EuGH - wie auch von der Europäischen Kommission in einem im Anschluss an das Urteil erlassenen „Factsheet“ hervorgehoben (http://ec.europa.eu/justice/data-protection/index_en.htm, Zugriff: 2.1.2016) - nicht geäußert (vgl. auch Weber/Heinrich 2014, Rz. 27 ff.).

5 Der britische Satiriker John Oliver nimmt diese Perspektive 2014 in seiner Sendung über das Recht auf Vergessen ins Visier: http://www.youtube.com/watch?v=r-ERajkMXw0 (Zugriff: 2.1.2016). 
zu beseitigen, generieren ihrerseits selbstverständlich Daten darüber, was Menschen gern verbergen lassen wollen. Es ist unschwer zu erkennen, dass gerade solche Daten von vielfältigem Interesse sind und leicht auch lukrativ genutzt werden können. ${ }^{6}$

\section{Hinweise auf die Geschichte eines Rechts auf Vergessen}

Das Urteil des EuGH ist in vielerlei Hinsicht brisant. Die Verwendung des Ausdrucks „Vergessen“ innerhalb der Rechtsprechung und die damit verbundene Vorstellung eines Rechts darauf, dass der Lauf der Zeit und damit des Vergessens unter gewissen Umständen nicht zum Nachteil einer Person aufgehalten werden dürfe, ist, jedenfalls innerhalb der schweizerischen Rechtsprechung, allerdings nicht neu. ${ }^{7}$ Hanspeter Thür, bis Ende 2015 Eidgenössischer Datenschutz- und Öffentlichkeitsbeauftragter, wies verschiedentlich darauf hin, dass im Rahmen eines Bundesgerichtsentscheides (BGE) bereits früher eine vergleichbare Formulierung Verwendung gefunden hatte. ${ }^{8}$ Es handelt sich dabei $\mathrm{u}$. a. um einen BGE von 1996. ${ }^{9}$ Darin geht es um die Frage, ob es sich bei einer Erwähnung der Verurteilung zu einer längeren Zuchthausstrafe in einem Zeitungsartikel 1993 um eine widerrechtliche Persönlichkeitsverletzung gehandelt habe oder nicht. Das Bundesgericht macht deutlich, dass es in solchen Fällen stets um eine Frage der Abwägung zwischen unterschiedlichen Interessen geht. Im konkreten Fall gibt das Bundesgericht der Privatperson, die eine Persönlichkeitsverletzung feststellen lassen wollte, Recht. Interessant ist dabei die Begründung:

Auf der einen Seite steht der Informationsauftrag der Beklagten [gemeint ist die Wochenzeitung; C. A.], zu dem es gehört, über ökonomische Zusammenhänge und dabei in Anbetracht der Wirtschaftslage über das Wirken von Unternehmenssanierern zu berichten (vgl. BGE 109 II 353 E. 3 S. 358 mit Hinweis). Sodann hat der Kläger - wie bereits erwähnt (E. 2c hiervor) - selber das Interesse der Öffentlichkeit an seiner geschäftlichen Tätigkeit geweckt,

6 Auf diesen Aspekt geht auch Nolte (2011) ein.

7 Für Überlegungen zum EuGH-Urteil und weiteren Rechtsfällen in Bezug auf ein „Recht auf Vergessen“ danke ich Herrn PD Dr. Michael Beusch, Universität Zürich, Richter am Bundesverwaltungsgericht St. Gallen.

8 So etwa im Rahmen einer Paneldiskussion zum Thema „Recht auf Vergessen im Internet zwischen Datenschutz und Zensur“, organisiert vom Europainstitut an der Universität Zürich am 13.11.2014. Entsprechendes findet sich auch bei Weber (2011), 121.

9 Vgl. BGE 122 III 449. 


\begin{abstract}
was eine Berichterstattung darüber nicht von vornherein als unrechtmässig erscheinen lässt. Schliesslich kann nicht als abwegig gelten, dass auch am Vorleben des Klägers ein gewisses öffentliches Interesse bestanden hat, ist er doch in einem Bereich tätig gewesen, der eine erhöhte Vertrauenswürdigkeit voraussetzte; verglichen mit bewilligungspflichtigen Berufen (z. B. Treuhänder, Wirtschaftsanwalt usw.) dürften auch wenig Zweifel daran bestehen, dass der Kläger angesichts seiner Vorstrafen wohl kaum je eine Zulassung erhalten hätte. Insgesamt erlaubt das von der Presse in Anspruch genommene Wächteramt, die Leserschaft vor fragwürdigem Geschäftsgebaren zu warnen. Stark ins Gewicht fällt auf der anderen Seite, dass die zu beurteilende Presseäusserung geeignet ist, das mit dem Strafvollzug verknüpfte Ziel der Resozialisierung zu vereiteln und zu verhindern, dass das dem normalen Lauf der Dinge entsprechende Vergessen eintreten kann (BGE 109 II 353 E. 3 S. 356; zum „Recht auf Vergessen“: BGE 111 II 209 E. [3]c S. 213 f.). Aufgrund der zeitlichen Distanz von rund zehn Jahren lässt sich unter diesem Blickwinkel ein überwiegendes Informationsinteresse nur noch schwer begründen [...]; vertreten wird gar, dass die Veröffentlichung einer gelöschten Vorstrafe stets unrechtmässig sei[.] ${ }^{10}$
\end{abstract}

Die Ausführungen sind in Bezug auf das Verständnis von Vergessen bemerkenswert. Eine Mitteilung einer Zeitung ist unter Umständen als Persönlichkeitsverletzung anzuerkennen, wenn dadurch „das dem normalen Lauf der Dinge entsprechende Vergessen“ nicht eintreten kann. Dessen Zeitdauer wird nachfolgend auf „rund zehn Jahre“ eingeschätzt. Aufgrund der vergangenen Zeitspanne scheint im konkreten Fall von 1996 ein legitimes öffentliches Informationsinteresse nur noch schwer kenntlich zu machen zu sein. Erwähnt wird im Entscheid des Bundesgerichts auch, dass vertreten wird, die Veröffentlichung einer gelöschten Vorstrafe sei in jedem Fall unrechtmäßig. Es wäre eine eigens zu behandelnde Frage, inwiefern diese Rechtsprechung auch auf die neuen Medien zu übertragen ist oder nicht. Hier ist zunächst einmal festzuhalten, dass der Rekurs auf ein Recht auf Vergessen innerhalb der jüngeren Rechtsprechung kein Novum darstellt. Sodann ist interessant, dass dieses Recht historisch betrachtet im Zusammenhang mit Fragen zu Kriterien für eine widerrechtliche Persönlichkeitsverletzung auftaucht. Während im eben dargelegten Fall von 1996 mit Verweis auf die gesellschaftlich erwünschte Resozialisierung einer Person das Recht auf Vergessen in die Urteilsfindung einbezogen ist, wird diesem Recht gut zehn Jahre früher in einem anderen Fall eine klare Absage erteilt. Es handelt sich dabei um die Klage von Robert Eibel gegen die Autoren des Buchs Die unheimlichen Patrioten. ${ }^{11}$ Im Urteil über Pressefreiheit und die Frage der Verletzung persönlicher Verhältnisse heißt es zusammenfassend: „Durch das Mittel der Druckerpresse verbreitete Äusserungen über die frühere politische Haltung von Personen der

10 BGE 122 III 449 E. 3b, S. 456/457.

11 Vgl. Frischknecht et al. (1984). 
Zeitgeschichte sind nicht widerrechtlich, sofern sie der Wahrheit entsprechen. Insoweit gibt es kein ,Recht auf Vergessen'“"12 Während also 1996 mit Verweis auf die gesellschaftlich erwünschte Resozialisierung eines ehemals Straffälligen dem Recht auf Vergessen stattgegeben wird, verneint das Gericht 1985 bei der Wahrheit entsprechenden Äußerungen betreffend die politische Gesinnung einer Person die Berufung auf dieses Recht. Das Bundesgericht entscheidet diesbezüglich anders als die beiden Vorinstanzen, die sich jeweils auf ein Recht auf Vergessen berufen hatten. Ausgehend von den beiden erwähnten Fällen bzw. den Urteilsbegründungen von 1996 und 1985 lässt sich bereits früh jene Bandbreite markieren, innerhalb derer Fragen bezüglich der Kriterien für ein Recht auf Vergessen auch heute noch kontrovers diskutiert werden.

\section{Das EuGH Urteil und das Recht des Einzelnen auf Vergessen}

Das Urteil, das im Frühjahr 2014 vom EuGH gefällt wird, bezieht deutlich Position bezüglich des Umgangs mit persönlichen Daten im Netz. Es unterstreicht das Recht des Einzelnen, auch im Web darüber mitzubestimmen, welche Informationen über eine Person aufgrund einer Suchanfrage mittels des Namens sofort zugänglich gemacht werden dürfen und welche nicht. Suchmaschinenanbieter haben Links zu von Dritten veröffentlichten Internetseiten mit Informationen zu einer Person zu entfernen, falls dies durch diese gefordert wird. Dies muss auch dann geschehen, wenn jemandem durch eine Information kein nachweisbarer Schaden zugefügt wird.

Art. 12 Buchst. b und Art. 14 Abs. 1 Buchst. a der Richtlinie 95/46[13] sind dahin auszulegen, dass im Rahmen der Beurteilung der Anwendungsvoraussetzungen dieser Bestimmungen u. a. zu prüfen ist, ob die betroffene Person ein Recht darauf hat, dass die Information über sie zum gegenwärtigen Zeitpunkt nicht mehr durch eine Ergebnisliste, die im Anschluss an eine anhand ihres Namens durchgeführte Suche angezeigt wird, mit ihrem Namen in Verbindung gebracht wird, wobei die Feststellung eines solchen Rechts nicht voraussetzt, dass der betroffenen Person durch die Einbeziehung der betreffenden Information in die Ergebnisliste ein Schaden entsteht. ${ }^{14}$

12 Vgl. BGE 111 II 209.

13 Es handelt sich dabei um die Richtlinie 95/46/EG des Europäischen Parlaments und Rates vom 24.10.1995 zum Schutz natürlicher Personen bei der Verarbeitung personenbezogener Daten und zum freien Datenverkehr, zu finden im EG-Amtsblatt, 23.11.1995, L 281/31 ff.

14 Vgl. EuGH-Urteil, Regeste (Tenor), Abschnitt 4. 
Unabhängig davon, ob eine Information richtig ist oder falsch und auch davon, ob jemandem durch Daten Schaden zukommt oder nicht, und schließlich unter Umständen auch ungeachtet dessen, ob die Information im Interesse einer Öffentlichkeit ist, müssen die Suchmaschinenanbieter gemäß Urteil auf Wunsch des Betroffenen hin Informationen entfernen, die bei einer Namenssuche erscheinen. ,Entfernen' bedeutet in diesem Zusammenhang - wie bereits erörtert - nicht, dass die Informationen vom Netz genommen werden. Allein die Verlinkung, aufgrund derer eine Information über eine Suche mittels eines Namens erscheint, muss gegebenenfalls aufgehoben werden. In der Regeste zum Urteil heißt es weiter:

\begin{abstract}
Da die betroffene Person in Anbetracht ihrer Grundrechte aus den Art. 7 und 8 der Charta verlangen kann, dass die betreffende Information der breiten Öffentlichkeit nicht mehr durch Einbeziehung in eine derartige Ergebnisliste zur Verfügung gestellt wird, überwiegen diese Rechte grundsätzlich nicht nur gegenüber dem wirtschaftlichen Interesse des Suchmaschinenbetreibers, sondern auch gegenüber dem Interesse der breiten Öffentlichkeit am Zugang zu der Information bei einer anhand des Namens der betroffenen Person durchgeführten Suche. ${ }^{15}$
\end{abstract}

Eine Einschränkung, die das Gericht diesbezüglich anführt, betrifft „,besondere Gründe“. Solche ergeben sich etwa in Bezug auf Personen, die ihrerseits als ,öffentliche' Personen gelten, bzw. Personen, die eine Rolle im öffentlichen Leben einnehmen und denen entsprechend ein größeres oder sogar überwiegendes öffentliches Interesse zukommt. In der erwähnten Regeste heißt es dazu: „Dies wäre jedoch nicht der Fall, wenn sich aus besonderen Gründen - wie der Rolle der betreffenden Person im öffentlichen Leben - ergeben sollte, dass der Eingriff in die Grundrechte dieser Person durch das überwiegende Interesse der breiten Öffentlichkeit daran, über die Einbeziehung in eine derartige Ergebnisliste Zugang zu der betreffenden Information zu haben, gerechtfertigt ist. " ${ }^{16}$ Das Urteil des EuGH widersetzt sich damit einem allfälligen Wunsch nach möglichst unmittelbarer, umfassender Transparenz und gewichtet in diesem Fall den Schutz der Privatsphäre und des Grundrechts des Einzelnen stärker als die Meinungsäußerungsfreiheit (Informationsfreiheit), stärker unter Umständen auch als etwa das Interesse einer kritischen Öffentlichkeit. Gleichzeitig lässt das Urteil durch den Hinweis auf „besondere Gründe“, die ein „überwiegendes Interesse der breiten Öffentlichkeit" rechtfertigen, aber doch auch einen Spielraum für Interpretation. Diese Formulierung verlangt nach einer jeweiligen Abwägung.

15 Vgl. ebd.

16 Vgl. ebd. 


\section{Wem kommt die Beweislast zu? Wer entscheidet?}

In einer Hinsicht kristallisiert sich im Urteil eine Perspektive, die im Kontext des Digitalen bislang wenig juristisches Gewicht erhalten hat. Es geht dabei um die Frage der Beweislast. Bislang lag diese auf Seiten des Betroffenen. Er musste klagen und vor Gericht deutlich machen, inwiefern eine zwar im Grunde richtige Information bei einer Suche nicht mehr erscheinen darf. Dabei musste der Kläger aufzeigen können, inwiefern ihm durch diese Information ein unangemessener Schaden entsteht. Das Gericht hatte dann darüber zu befinden, ob dieser Schaden tatsächlich als unangemessen zu gelten hat oder aber vertret- und zumutbar ist. Dies ist gemäß EuGH-Urteil in Bezug auf die Angaben bei einer Namenssuche innerhalb der EU seit Mai 2014 nicht mehr so. Bei Zweifeln daran, dass Informationen bei der Eingabe eines Namens unmittelbar und sofort an erster Stelle ersichtlich erscheinen dürfen, liegt die Beweislast fortan bei den Suchmaschinenanbietern, die zu einer Art Vertreter der kritischen Öffentlichkeit generieren, die, hypothetisch jedenfalls, möglichst ungehindert alle Informationen erhalten möchte. ${ }^{17}$ Die Suchmaschinenanbieter müssen eine Rechtfertigung vorbringen, falls sie sich weigern, eine Information trotz Ersuchens eines Betroffenen zu entfernen. Bei einer Zurückweisung eines Antrags kann der Betroffene gegen Google ${ }^{18}$ klagen und den Entscheid somit an ein Gericht ziehen. Dieses hat dann darüber zu befinden, ob Google das Urteil des EuGH richtig umgesetzt hat oder nicht. In der Rechtfertigungsposition befindet sich in diesem Szenario der Vertreter des Suchmaschinenanbieters. ${ }^{19}$

Seit dem Urteilsspruch des EuGH sind in der Schweiz laut dem Transparenzbericht von Google ${ }^{20}$ bis Januar 2016 mehr als 8600 Anfragen bezüglich einer

17 Diese Formulierung verschleiert ihrerseits, dass Google stets und weiterhin unverändert nach nicht vollständig transparenten Algorithmen die Informationen strukturiert, welche bei einer Namenssuche erscheinen. Vgl. dazu auch die Informationen zu den sogenannten ,Echoräumen` in Ryan (2013).

18 Hier und im Folgenden wird von Google und den Zahlen, die von Google veröffentlicht werden, ausgegangen. Es sind jedoch Suchmaschinenanbieter in den EFTA-, den EWR-, und den EU-Staaten von dem Urteil betroffen bzw. setzen es seit Frühling 2014 um.

19 Zur insoweit vergleichbaren Situation bei der Gewährleistung des Persönlichkeitsschutzes bei der Publikation von Personendaten in Google Street View vgl. BGE 138 II 346 E. 10.6.3 u. 14.4. Danach muss Google auf Anzeige von Betroffenen hin manuell hinreichende Anonymisierungen in Street View vornehmen, die die Anonymisierungssoftware nicht automatisch ausführte. Zu Instrumenten zur Gewährleistung des grundrechtlichen Datenschutzes, der seinerseits Teil des u. a. durch Art. 13 Abs. 2 der Bundesverfassung geschützten Rechts auf informationelle Selbstbestimmung ist, vgl. (auch unter Bezugnahme auf den „Google-Spain“-Entscheid des EuGH) Schweizer (2014), Art. 13 Rz. 85 ff.

$20 \mathrm{Vgl.http://www.google.com/transparencyreport/removals/europeprivacy/} \mathrm{(Zugriff:} \mathrm{2.1.2016).}$ 
Löschung von Links eingegangen. Frankreich und Deutschland stehen hinsichtlich der Anzahl gestellter Löschgesuche an erster Stelle. Aufgrund des EuGH-Urteils und der veränderten Beweislast kommt Google damit eine nicht angestrebte, aber unübersehbar noch einmal wichtigere Stellung bezüglich Datenvermittlung im Netz zu. Die Suchanbieter müssen nun Kriterien darüber erarbeiten, aufgrund derer die Anfragen beurteilt und gutgeheißen oder abgelehnt werden. Bei Google heisst es dazu: „Bei allen diesen Ersuchen müssen wir das Recht des Einzelnen auf Vergessenwerden und das Recht der Öffentlichkeit auf Information gegeneinander abwägen - individuell und fallbasiert. Es ist uns wichtig, die richtige Abwägung zu treffen. Diese Verpflichtung stellt uns vor ganz neue und schwierige Herausforderungen und wir sind auf der Suche nach Unterstützung bei der Frage, welche Prinzipien Google bei fallbasierten Entscheidungen anwenden sollte. Aus diesem Grund haben wir einen Experten-Beirat einberufen. “21

Auf der Site von Google, auf der der Transparenzbericht aufgeschaltet ist, gibt das Unternehmen zudem Beispiele an, die Einblick vermitteln, wie Google im einzelnen Fall entschieden hat. In einer Art anonymisierten Kasuistik bietet Google so Einsicht in die vom Großunternehmen vorgenommene Entscheidungsfindung. Die Suchmaschinenanbieter sind durch das EuGH in eine Rolle katapultiert, die der einer juristischen Instanz durchaus nahe kommt. ${ }^{22}$

\section{Formen von Vergessen: Zur Unterscheidung zwischen Information und Mitteilung}

Parallel zur Durchsetzung der Digitalisierung lässt sich ein deutlich verstärktes Interesse an Formen von Vergessen feststellen. ${ }^{23}$ Dies hat verschiedene Gründe. Eine zentrale Rolle spielt dabei die Digitalisierung selbst, verstanden als ein kommunikatives System, das über ein vorher nicht gekanntes Potential an Daten-

21 https://www.google.com/intl/de/advisorycouncil (Zugriff: 2.1.2016). Die zehn Experten des Beirats stellt Google vor; vgl. https://www.google.com/intl/de/advisorycouncil/ (Zugriff: 2.1.2016).

22 Wie bereits erwähnt, hat im Streitfall ein Gericht darüber zu befinden, ob Google das Gesetz richtig umsetzt oder nicht. Google ist ein privates Unternehmen, gegen welches vor einem staatlichen Gericht Klage erhoben werden kann.

23 Ein bekanntes Beispiel hierzu ist etwa Mayer-Schönberger (2010, der vorschlägt, jeden Inhalt im Netz beim Abspeichern mit einem Laufzeitdatum zu versehen. Diese Idee wird in verschiedenen jüngeren Publikationen aufgenommen und diskutiert. Auf die Schwächen des sogenannten „digitalen Radiergummis“ geht u. a. Nolte (2011) ein. 
sammlung, Datenspeicherung und Datenverknüpfung verfügt. Elena Esposito spricht in diesem Zusammenhang von der Herausbildung eines „telematischen Gedächtnisses“24. Dieses ist dem menschlichen in einer Hinsicht offensichtlich weit überlegen: Unter dem Eindruck der immensen Datensammlungskapazität erscheint die Fähigkeit des Menschen, Inhalte zu lernen und diese später erinnernd abrufen zu können, kaum mehr besonders interessant. Hingegen wird das Vergessen, verstanden als das Vermögen, Selektionen vorzunehmen und zwischen unterschiedlichen Inhalten zu priorisieren, zum Faszinosum, zu einer Fähigkeit, über die Technik mit künstlicher Intelligenz erst teilweise verfügt. Unter dem Eindruck des telematischen Gedächtnisses erscheint nicht mehr das Erinnern, verstanden als Speichern und Abrufen, anspruchsvoll, sondern der gezielte Einsatz von Formen von Vergessen. „Vergessen“ bedeutet dabei das komplexe Vermögen, zwischen verschiedenen vorhandenen „Daten“ zu differenzieren. In Anbetracht der Digitalisierung aller Lebensbereiche und der unüberblickbar anwachsenden Menge an gesammelten Daten ist damit nicht das Verfügen über Daten, sondern das Unterscheiden von wichtigen und unwichtigen Daten eine eminent zentrale Kompetenz.

Bei der Transformation, die unter den Zeichen der Digitalisierung besonders interessiert, handelt es sich entsprechend nicht um das Abspeichern von Informationen, sondern um das Umwandeln von Informationen in Mitteilungen. Diese vollzieht sich notwendigerweise durch den Einsatz von Formen von Vergegenwärtigen und Vergessen. Die Bedeutung des Begriffs „Vergessen“ erfährt unter den Zeichen des Digitalen eine spezifische Prägung. Vergessen ist in diesem Kontext nicht als das Gegenteil von Erinnern zu begreifen, sondern als eine Bedingung und eine Wechselwirkung davon.

Es war zwar im 20. Jahrhundert weitherum gebräuchlich, Vergessen und Erinnern von ihrer Bedeutung her als Gegensätze vorzustellen, wobei das Erinnern mit Hervorholen-Können und das Vergessen mit Verloren-Haben in Verbindung gebracht wurde. Diese Vorstellung lässt sich aber kaum aufrecht halten. ${ }^{25}$ Die Prozesse von Erinnern und Vergessen stehen vielmehr in einem engen, sogar symbiotischen Verhältnis zueinander. Um etwas zu erinnern, zu vergegenwärtigen, müssen unzählige andere Wahrnehmungen und Denkmöglichkeiten ausgeblendet werden können. Vergessen ist so gesehen die Voraussetzung dafür, dass man sich auf etwas Bestimmtes konzentriert einlassen kann. In dem Moment, in dem eine Information erfasst werden will, wird diese aktualisiert und so zu einer Mitteilung. Diese Aktualisierung vollzieht sich durch unzählige Vergessensvorgänge,

24 Esposito (2002).

25 Zur jüngeren Begriffsgeschichte von „Vergessen“ und „Erinnern“ vgl. Abbt (2016). 
die erst ermöglichen, dass aus der Fülle an Informationen jenes Besondere, das jemand aufnehmen und verstehen möchte, begreifbar wird. Vergessen bedeutet also nicht das totale Löschen, sondern das vorübergehende Ein- oder Ausblenden von Informationen. Vergessen ist im Gang, damit die Fülle an Informationen einerseits vernachlässigt und damit andererseits bestimmte Informationen aktualisiert und so zu Mitteilungen transformiert werden können.

Es ist diese Verwendung des Begriffs „Vergessen“, die im hier im Fokus stehenden EuGH-Urteil in Bezug auf das „Recht, vergessen zu werden“ zur Anwendung kommt. Das Urteil veranlasst nicht dazu, gewisse Daten zu löschen, sondern dazu, bestimmte Daten so zu platzieren, dass sie weniger leicht zu Mitteilungen mit öffentlicher Wirkung werden. Die Richter und Richterinnen widersprechen damit deutlich einer gängigen Vorstellung im Kontext des Digitalen. Diese lautet: „Das Netz vergisst nicht!“26 In dieser Aussage drückt sich eine weitverbreitete Vorstellung aus, wonach es im Netz kein Vergessen und also keine Evaluationsprozesse gebe. Es drückt sich darin etwas aus, was Phantasma des Digitalen genannt werden kann. Dieses Phantasma gleicht dem Trugbild, das Michel Foucault 1970 in Bezug auf die Bibliotheken und deren soziale Bedeutung diagnostizierte. Foucault spricht in den Schriften zur Literatur von einer „,fantastique“ de bibliothèque“ ${ }^{\text {“27. }}$. Die Idealvorstellung sei so wirksam, so Foucault, dass dabei übersehen werde, dass Bibliotheken, Enzyklopädien und Archive die Gesamtheit des tradierten Wissens stets und im besten Falle nur als Potentialität zur Verfügung stellt. Stets bleibt diese Potentialität auf Strukturierung und auf Aktualisierung angewiesen. Bei den Bibliotheken waren und sind etwa die Kataloge strukturierende Elemente, die einem Suchenden helfen, das richtige Schriftstück zu finden. Wenn aber kein Interesse da ist, hilft auch die Strukturierung des Wissens nur wenig, damit aus Informationen Mitteilungen werden. Voraussetzung dafür ist an zumindest irgendeiner Stelle ein kritisches Bewusstsein, das nach Informationen fragt und sucht. Was Foucault über die Bibliothek und das Archiv ausführt, lässt sich auch auf das Netz übertragen. Erst Strukturierung und Aktualisierung transformieren Informationen zu Mitteilungen. In beiden Fällen vollziehen sich die Transformationen durch Ausschlussmechanismen, durch Priorisierung und Selektion, durch Formen von Vergessen. Ohne jede Art von Evaluationsprozess ist Erinnerung ein leerer Begriff.

Das digitale Netz ist zwar nicht nur eine passive Datensammlung, sondern auch eigens ein dynamisches Kommunikationssystem, aber es bleibt auf eine

26 Gibt man in eine Suchmaschine des Netzes ein „Das Netz vergisst nicht(s)“, dann erscheinen mehr als 218000 Verweise. Viele Seiten, die diese Äußerung beinhalten, bestätigen auch die damit gemachte Aussage. Dagegen hält Kutter (2012).

27 Foucault (1988). Vgl. zu Foucaults Überlegungen auch Pethes (2008), 106-125. 
Kommunikation mit anderen Systemen und auch auf eine Kommunikation mit Menschen ausgerichtet. Es ist nicht möglich, alles, was gespeichert wird, bei einer Suchanfrage gleichzeitig nebeneinander erscheinen zu lassen. Es sind viele und für den Einzelnen oft undurchsichtige Auswahlmechanismen mit im Spiel, die $\mathrm{zu}$ einer bestimmten Zusammenstellung von Informationen, etwa bei einer Suche im Netz, führen.

Die Vorstellung, dass im sogenannten „telematischen“ Gedächtnis nichts vergessen gehe, ist deshalb irreführend. Selektionsprozesse finden im Netz fortlaufend und in hohem Maße statt; es fragt sich nur, nach welchen Kriterien.

\section{Der Faktor Zeit und die Frage der Verjährung in Recht und Ethik}

Die Entscheidungsträger bei den Firmen der Suchmaschinenanbieter haben heute konkret je zwischen einerseits dem Interesse der Öffentlichkeit und der Informationsfreiheit, die es innerhalb des Netzes zu erhalten oder zu befördern gilt, und andererseits dem Recht auf Vergessenwerden, das einer Person im Umgang mit Daten zusteht, abzuwägen. Dabei betrifft ein Kriterium, das Google für die Entscheidungsfindung als relevant nennt, die Aktualität und Bedeutung einer Information für die gegenwärtige ,kritische‘ Öffentlichkeit. Tatsächlich betreffen viele Löschanträge Inhalte, die nicht nur richtig und zutreffend sind, sondern gegen die zu einem gewissen Zeitpunkt $x$ auch nichts vorgebracht worden ist, auch nicht von der involvierten Person. Manchmal werden Löschanträge gestellt, die Daten betreffen, die von jemandem sogar selbst ins Netz gestellt und zum Zeitpunkt $x$ noch als unproblematisch eingestuft worden waren. Zum Zeitpunkt $x+10$ Jahre aber erscheint derselbe Inhalt unter Umständen als nicht mehr adäquat. Die Frage danach, wie lange Daten - gesellschaftlich und/oder individuell - Bedeutung haben (und haben sollen), ist nicht leicht allgemein zu beantworten. Eine Abwägung im Einzelfall ist diesbezüglich unabdingbar. Gleichzeitig gibt es einen breiten Konsens darüber, dass innerhalb des Netzes dem Ablauf der Zeit und der Korrelation zwischen Zeit und Relevanz bei Informationen keine oder zu wenig Rechnung getragen wird..$^{28}$ Das Recht auf Vergessen kann entsprechend auch als ein Recht formuliert werden, das erlaubt, den Faktor Zeit miteinzubeziehen.

28 Auf dieses Defizit weist u. a. Hanspeter Kellermüller, Generalsekretär der NZZ-Mediengruppe, im Rahmen der Tagung Haftung von Medien im Internetzeitalter hin, Bern, 11.9.2013. Viktor Meyer-Schönberger schlägt seinerseits zur Behebung dieses Problems vor, jeden Inhalt im Netz beim 
Die Vorstellung der Möglichkeit der Verjährung ist innerhalb der abendländischen Rechtskultur historisch tief verankert. ${ }^{29}$ Das Institut ist auch innerhalb des Strafrechts zentral. Es zeichnet den demokratisch verfassten Rechtsstaat aus, dass darin sowohl Delinquente nach einer gewissen Zeit unter Umständen wieder eine zweite Chance innerhalb der Gesellschaft erhalten ${ }^{30}$ als auch Delikte verjähren und also strafrechtlich unter Umständen nicht mehr verfolgt werden können. Eine Ausnahme dazu bilden u. a. Verbrechen wider die Menschlichkeit, bei denen davon ausgegangen wird, dass die Zeit keine abschwächende Wirkung zu entfalten vermag. ${ }^{31}$ Gerade diese auch in der Gesetzgebung der Schweiz angelegten Ausnahmen zeigen an, wie grundlegend der Zusammenhang von Straftat, Strafmass und Temporalität in Hinsicht auf Fragen der Gerechtigkeit ist. ${ }^{32}$ Die Diskussionen über ein Recht auf Vergessen rücken das Augenmerk verstärkt auf diesen zentralen Zusammenhang und fragen danach, wie der juristische Grundsatz der Verjährbarkeit auch innerhalb des Internets ernst genommen und umgesetzt werden kann.

Die juristische Diskussion über Verjährung und die Möglichkeit einer zweiten Chance sind in einen ethischen Horizont eingebettet. Die Vorstellung, dass sich der Mensch verändern kann, dass er lernfähig ist und sich willentlich entwickeln kann, prägt das philosophische Denken seit der Antike. ${ }^{33}$ Eine besondere Gewichtung der Eigenschaft des Menschen, sich gestalten und aus der Geschichte lernen zu können, wird von verschiedenen Philosophen der Aufklärung vorgenommen. Sie prägen den Begriff der Perfektibilität des Menschen. Perfektibilität bedeutet, dass der Einzelne sich durch Einsicht, Übung, Erfahrung und Disziplin weiter entwickeln und vervollkommnen kann. ${ }^{34}$ Dieses Vermögen wird dabei nicht nur als Bedingung für die Pädagogik ernst genommen, sondern es wird grundlegend

Abspeichern mit einem Laufzeitdatum zu versehen; vgl. Mayer-Schönberger (2010). Diese Idee wird in verschiedenen jüngeren Publikationen aufgenommen und diskutiert. Auf die Schwächen des sogenannten „digitalen Radiergummis“ geht u. a. Nolte (2011) ein.

29 Vgl. Oetker (1994).

30 Die Eidgenössische Initiative Lebenslange Verwahrung für nicht therapierbare, extrem gefährliche Sexual- und Gewaltstraftäter, die in der Schweiz von Volk und Ständen am 8.2.2004 angenommen wurde, ist $\mathrm{u}$. a. auch deshalb unter Gesichtspunkten der Gerechtigkeit problematisch, weil sie zum Ziel hat, den Grundsatz der Überprüfbarkeit eines Urteils im Laufe der Zeit und unter Inrechnungstellung von Veränderungen in der Zeit aufzuheben.

31 Vgl. Art. 101 Abs. 1 des Schweizerischen Strafgesetzbuches vom 21.12.1937 (Systematische Rechtssammlung des Bundesrechts 311.0).

32 Vgl. dazu u. a. Schefczyk (2012).

33 Zur Idee der Perfektibilität, die bereits im aristotelischen Prozessmodell angelegt sei, vgl. Maaßen (2015), $274 \mathrm{ff}$.

34 Vgl. Henning (2015). 
für die Analyse aller Lebensbereiche, insbesondere der Sittenlehre und Ethik. Unter dieser Perspektive ist der Einzelne nicht in erster Linie aufgrund seiner Geschichte, Herkunft oder Vergangenheit zu beurteilen, sondern aufgrund seiner Verhaltensweise und seinem Bemühen in der Gegenwart. Immer ist dem Anderen nämlich zuerst einmal zu unterstellen, so der Vorschlag $\mathrm{u}$. a. von Immanuel Kant, er könne sich verändern, sich bestimmen und vor allem: aus Fehlern lernen. Von dieser Annahme ist prinzipiell nicht abzukommen, auch wenn die gegenwärtige Erfahrung das Gegenteil offenbart und dann nach einer Sanktion verlangt, die ihrerseits allerdings die Potentialität einer positiven Veränderung in Rechnung nimmt. $^{35}$

Aus diesem in der Aufklärung nachhaltig installierten Menschenbild resultiert nicht nur die Überzeugung, dass im Zweifel stets für den Angeklagten Partei zu nehmen sei, sondern auch die Überzeugung, dass ein Mensch zu Lebzeiten nicht und nie definitiv und abschließend beurteilt werden könne und dürfe. Nach der realisierten Sanktion, nach der Übernahme von Verantwortung ist jeder Person grundsätzlich wieder eine neue Chance zuzusprechen. Es liegt sogar im Interesse der gesamten Gesellschaft und im Interesse jedes Einzelnen, dass alles daran gesetzt wird, möglichst jeden Menschen stets wieder in die Gesellschaft $\mathrm{zu}$ integrieren, ihm eine neue Chance zu geben. Die Begründungen für diese Haltung sind unterschiedlich. Es geht dabei in den Argumentationen der Aufklärer und der frühen liberalen Denker selten nur um den Täter, nur um die Hilfe desjenigen, der eine Verfehlung vorgenommen hat. Im Vordergrund steht die Vorstellung, dass es zum Schutze und Vorteil aller sei, dass niemand etwas so Schlimmes tun könne, dass er sein Menschsein ganz und gar veräußert. ${ }^{36}$ In Recht und Ethik kommt nicht zuletzt daher dem Ziel der Resozialisierung eine zentrale Bedeutung zu.

\section{Legitime Resozialisierungsbemühung oder ungerechtfertigter Anspruch auf Vergessen?}

Im juristischen Kontext gilt Resozialisierung in der Schweiz seit Inkrafttreten des Strafgesetzbuchs von 1942 als Ziel verhängter Strafen und Maßnahmen. Der Begriff kommt im Gesetz allerdings nicht vor. In seiner bis Ende 2006 geltenden Fassung sah der damalige Art. 37 vor, „der Vollzug solle erziehend auf den Gefan-

35 Vgl. dazu Guyer (2009).

36 Vgl. dazu u. a. Schaber (2012). 
genen einwirken und ihn auf den Wiedereintritt in das bürgerliche Leben vorbereiten“. Die Formulierung, dass eine erwachsene Person durch den Aufenthalt in einer geschlossenen Institution ,erzogen' werden solle, wird heute weitherum als inakkurat aufgefasst. Peter Aebersold hält dazu bereits zum alten Recht fest: „Diese Formulierung gilt schon länger als veraltet, weil wir es als überheblich empfinden, Erwachsene erziehen $\mathrm{zu}$ wollen. Zudem ist unbestritten, dass die Beeinflussung nur auf Legalbewährung, nicht aber auf eine moralische Besserung abzielen darf. Statt der ,erzieherischen' Einwirkung hat sich in Theorie und Praxis der Begriff Resozialisierung durchgesetzt.“37 Resozialisierung zielt ab auf eine „Legalbewährung“, das heißt, auf einen Zustand - sowohl psychisch, als auch physisch und materiell -, in dem ein Betroffener die Möglichkeit hat, sein Leben innerhalb der Legalität selbständig zu führen. ${ }^{38}$ Dieses Ziel des Strafvollzugs ist in den letzten Jahren aus unterschiedlichen Gründen und von verschiedenen Seiten unter Druck geraten. ${ }^{39}$ Gleichwohl gilt Resozialisierung nach wie vor als Kernanliegen des Maßnahmenkatalogs innerhalb des schweizerischen Strafvollzugs. ${ }^{40}$

Erfolgreiche Resozialisierungsprozesse führen zu einer Wiedereingliederung einer Person in das soziale Gefüge der Gesellschaft. ${ }^{41}$ Damit dies möglich wird, sind nicht nur Aufarbeitungs- und Erinnerungsprozesse, sondern oft auch vielfältige Formen von Vergessen am Werk. Jene Form, die hier in Bezug auf das EuGHUrteil vom Mai 2014 interessiert, ist eine Form von Vergessen, die einer Person konkret eine zweite Chance ermöglichen kann.

Es ist denkbar, dass in einem konkreten Fall Resozialisierung nur realistisch umgesetzt werden kann, wenn eine Information über eine Person nicht unmittelbar allen zugänglich ist. ${ }^{42}$ Aus der Perspektive einer Ethik der zweiten Chance

37 Aebersold (2009).

38 So spricht Art. 75 StGB in seiner heutigen Version davon, der Strafvollzug habe das soziale Verhalten des Gefangenen zu fördern, insbesondere die Fähigkeit, straffrei zu leben (Abs. 1) sowie davon, der Gefangene habe bei den Sozialisierungsbemühungen und den Entlassungsvorbereitungen aktiv mitzuwirken (Abs. 5); vgl. dazu etwa Brägger (2013), Art. 75 Rz. 1 ff., 11.

39 Verschiedene Abstimmungsvorlagen schlagen vor, Resozialisierung als Ziel von Strafen und Massnahmen abzuschwächen oder aufzuheben.

40 Vgl. etwa anstelle zahlreicher BGE 133 I 270 E. 3.2.2, der ausdrücklich von einem öffentlichen Interesse an einer Resozialisierung spricht.

41 Vgl. Cornel (2009), 27-59; zum Recht der Resozialisierung in Deutschland auch ders. (2012). 42 Mit der Kenntnis der Vergangenheit dieser Person im rechtlichen Umfeld befasst sich das Strafregister (Art. 365 ff. StGB). Aus dieser dem amtlichen Erinnern dienenden Datensammlung werden die Einträge nach einer nach Schwere des zur Eintragung führenden Deliktes abgestuften Dauer von Amtes wegen entfernt, sprich physisch eliminiert (Art. 369 Abs. 1-6 StGB). Die Eintragung darf nicht mehr rekonstruierbar sein und das entfernte Urteil darf dem Betroffe- 
ist der Anspruch auf das Recht, vergessen zu werden, wie es das EuGH-Urteil vorsieht, in einem solchen Fall legitim. Das Recht auf diese Art von Vergessen umfasst zum Beispiel das Recht, nach geleistetem Strafvollzug die Chance zu erhalten, noch einmal neu anzufangen. Es kommt auch dann zum Tragen, wenn eine Person in einer Hinsicht gescheitert ist und nach Ablauf einiger Jahre glaubhaft einen Neuanfang bewerkstelligt oder bewerkstelligen möchte. Diese Form von Vergessen kann aber nicht nur gerechtfertigt von Personen geltend gemacht werden, die in irgendeiner Weise negativ aufgefallen sind, sondern auch von Seiten eines Opfers, das nicht möchte, dass Informationen über erlebtes Unrecht auch noch nach Jahren stets unmittelbar allen leicht zugänglich sind. Wer das Recht auf Vergessen geltend macht, ist in diesen Fällen daran interessiert - möglichst ohne Aufsehen zu erregen -, das eigene Leben innerhalb des sozialen Gefüges der Gesellschaft zu bewältigen und so weit als möglich frei zu gestalten. Es handelt sich dabei um die Forderung, Informationen vergessen zu machen, die bereits einmal sozial zur Mitteilung geworden waren oder noch Mitteilung sind. Das Urteil über ein Recht, vergessen zu werden, wie es vom EuGH gefällt und formuliert wurde, zielt meines Erachtens auf solche Fälle ab. Es geht dabei darum, einem Menschen innerhalb der Gesellschaft nicht nur moralisch eine zweite Chance zuzugestehen, sondern darum, dem Einzelnen das Recht zuzusprechen, diese zweite Chance gesellschaftlich, hier insbesondere im Rahmen des Digitalen, durchzusetzen.

nen nicht mehr entgegengehalten werden (Art. 369 Abs. 7 StGB). Konsequenterweise sind die Strafregisterdaten auch nicht zu archivieren (Art. 369 Abs. 8 StGB). Bereits zu einem früheren Zeitpunkt werden Einträge nicht mehr in die von Privatpersonen angeforderten, sie selbst betreffenden Strafregisterauszüge aufgenommen (Art. 371 Abs. 3 StGB). Derartige Auszüge werden nicht selten bei Stellenbewerbungen durch den die Stelle ausschreibenden Arbeitgeber verlangt. Der Umstand, dass in solchen Konstellationen in den vom Bewerber angeforderten und hernach beim potentiellen Arbeitgeber einzureichenden Privatauszügen schon früher keine Einträge mehr aufscheinen als im amtlichen Verkehr, entspringt einer bewussten Wertung des Gesetzgebers, der damit die Resozialisierung nicht behindern will (vgl. dazu etwa Trechsel/Lieber 2013, Art. 371 Rz. 4). Das System wird durch den Bundesrat in seiner seinerzeitigen Botschaft an den Gesetzgeber zu Art. 369 Abs. 7 [entsprechend dem damaligen Art. 372 Abs. 7 in Entwurfform] treffend beschrieben: „Artikel 372 Absatz 7 E ordnet ausdrücklich an, dass entfernte Daten nicht mehr rekonstruierbar sein dürfen. Der zweite Satz bezeichnet die eigentliche rechtliche Wirkung der Entfernung der Eintragung: Das betreffende Urteil und damit auch die Tat selbst dürfen dem Täter nicht mehr entgegengehalten werden, das heisst, es dürfen daran keine Rechtsfolgen mehr geknüpft werden. Der Täter ist vollständig rehabilitiert. Im privaten Verkehr darf sich der Betroffene allerdings schon als nicht vorbestraft bezeichnen, wenn der Strafregisterauszug keinen Eintrag mehr aufweist“; Botschaft vom 21.9.1988 zur Änderung des Schweizerischen Strafgesetzbuches, Bundesblatt [BBl] 19992168. 
Um eine andere Form von Vergessen, die bisweilen eingefordert wird, handelt es sich dagegen bei dem Wunsch, Informationen gar nicht erst publik werden zu lassen. Wer das Recht auf Vergessen als ein Recht auf Unterdrückung von Informationen nutzen möchte, dem geht es nicht um eine Art Unterschlupf in der gesellschaftlichen Normalität, sondern um ein Verbergen von Informationen, die für ihn selbst unangenehm sind, gleichzeitig aber von Interesse für andere. So nachvollziehbar der Wunsch nach dem Verbergen von Peinlichkeiten oder nach Erhalt der gesellschaftlichen Anerkennung im Einzelfall sein kann, so schwierig ist es, diesen Anspruch als einen ethischen oder einen gesetzlichen und damit staatlich durchsetzbaren zu rechtfertigen.

Die Unterscheidung in Informationen auf der einen und Mitteilungen auf der anderen Seite ist hilfreich für die Differenzierung der unterschiedlichen Ansprüche auf Formen von Vergessen. Erstere Form, die hier als legitime vorgstellt wird, fragt nach der Abschwächung einer Mitteilung, die bereits öffentlich geworden war oder ist. Eine Handlung wurde bereits öffentlich verhandelt, sie hat bereits Wellen geschlagen, Konsequenzen nach sich gezogen. Nun geht es darum, die Wirksamkeit dieser Mitteilung abzuschwächen und Vergessensprozesse in Gang zu bringen, damit jemandem die Möglichkeit geschaffen wird, sein Leben noch einmal neu und konstruktiv einzurichten. Im zweiten Fall geht es darum, eine Information gar nicht erst zur Mitteilung werden $\mathrm{zu}$ lassen. Im strengen Sinn geht es dabei nicht um die Frage des Faktors Zeit. Es stellt sich nicht die Frage danach, wie lange jemand durch eine Mitteilung in seinem Lebensvollzug gehindert werden darf, sondern um die Frage, ob durch eine Mitteilung jemand überhaupt erst in ein neues Licht gerückt werden darf. Wer in dieser Art auf ein Recht auf Vergessen pocht, fordert im Grunde für sich selbst ein Recht auf Zensur ein.

Der Wunsch nach legitimer Resozialisierungsbemühung und jener nach ungerechtfertigtem Streben nach Zensur sind nicht in jedem Fall klar und eindeutig auseinanderzuhalten. Grenzfälle sind denkbar. Wenn Vergessen aber als ein Abschwächen von wirksamen Mitteilungen verstanden wird, als eine Transformation einer Mitteilung zu einer Information, dann lassen sich Formen von Zensur deutlich davon unterscheiden. Mit zwei dazu einschlägigen Beispielen lässt sich das bereits Erläuterte weiter vertiefen und in Bezug auf die Frage, wann ein Recht auf Vergessen im Kontext des Digitalen ${ }^{43}$ begrüßenswert sein kann und wann nicht, eine Orientierung erzielen.

In Bezug auf das Recht, zugunsten einer gelingenden Resozialisierung vergessen zu werden, bietet sich als Beispiel der straffällige Kinderschänder an, der

$43 \mathrm{Zu}$ „Ubiquität und Entgrenzung“ als Merkmale von Digitalisierung vgl. Boehme Nessler (2008), 102-104. 
durch die Institutionen gegangen ist, alle gesetzlich verhängten Strafen verbüßt hat und nach Jahren der Haft in wiedererlangter Freiheit versucht, sozial Fuß zu fassen. In diesem Fall kann der Anspruch auf ein Recht auf Vergessen, wie es durch das EuGH am 13. Mai 2014 bekräftigt worden ist, durchaus Sinn ergeben. Erscheint bei einer Namenssuche unmittelbar stets die Darstellung des seinerzeit begangenen Verbrechens, das Urteil und allenfalls auch Medienberichte und vielleicht sogar weitere Einträge, die die Person bezichtigen, so kann eine Resozialisierung zu einem kaum erreichbaren Ziel werden. Weder Wohnung noch eine Arbeitsstelle wird diese Person finden. Auch der keine (Vor-)Strafe mehr aufweisende Strafregisterauszug nützt dann diesbezüglich nichts. In diesem nur schemenhaft skizzierten Fall ist der Einzelne auf den Schutz des Staates angewiesen. Von staatlicher Seite muss dafür gesorgt bleiben, dass die Grundrechte auch dieser Person nicht verletzt werden, und das heißt, dass diese Person innerhalb des gesetzlichen Rahmens unbehelligt ihren Alltag bewältigen und gestalten kann und darf. ${ }^{44}$ Es ist verständlich und richtig, dass diese Person sich auf das Recht auf Vergessen berufen kann.

Nicht angemessen ist dagegen der Rekurs auf das Recht, vergessen zu werden, im folgenden Fall. Das Beispiel eignet sich, herangezogen zu werden, da die involvierte Person ihrerseits explizit das Recht auf Vergessen in die Diskussion einbringt. Es handelt sich dabei um eine Schweizer Politikerin, die innerhalb des gesetzlichen Rahmens Steueroptimierungen vorgenommen hat. Da eben diese sich seit Jahren gegen die Pauschalbesteuerung ausspricht, ist dieses Verhalten nicht ohne Brisanz. Die Frage, ob das, was legal ist, auch legitim sei, wird von den Medien aufgegriffen. Unter der Überschrift „Wasser predigen und Wein trinken“ behandelt etwa die NZZ die Diskrepanz zwischen politischer Theorie und persönlicher Praxis in besagtem Fall, ${ }^{45}$ der, am 5.11.2014 zuerst von der Weltwoche aufgebracht, ab Mitte November 2014 in der deutschsprachigen Schweiz zu einer Angelegenheit des öffentlichen Interesses heranwächst. Für die hier im Zentrum stehende Thematik ist der Fall interessant, weil, wie erwähnt, die betroffene Politikerin in einem Interview im Schweizer Fernsehen für sich das Recht auf Vergessen beansprucht. Das Vergessen wird dabei in Bezug auf einen Sachverhalt von 2005 eingefordert. Die Journalistin, die das besagte Interview führt, deutet in ihrer Frage an, dass es sich bei dem aktuellen und bei dem früheren Sachverhalt allenfalls um vergleichbare Verhaltensweisen handle: „Sie stehen nun zum zweiten Mal wegen finanzieller Fragen im Fokus. Vor einigen Jahren wurde Ihnen in der Mieter-

44 Zum Schutz des Privaten im Zeitalter des Digitalen und der staatlichen Verpflichtung vgl. diesbezüglich Lindner (2014) u. McArthur (2001).

45 Häfliger (2014). 
verbands-Affäre vorgeworfen, Sie hätten Mietern unrechtmäßige Kosten verrechnet. Sind Sie politisch noch tragbar?“ Die Politikerin antwortet: „Für diesen einen Mieterwechsel beanspruche ich das Recht auf Vergessen. Wenn das heute noch thematisiert wird, ist das eine Persönlichkeitsverletzung. Die Angaben zu diesem Sachverhalt von 2005, die noch im Internet zirkulieren, sind nicht gerichtsfest. “46 Die in der Öffentlichkeit stehende Person beruft sich also auf ein Recht auf Vergessen und weicht mit diesem Verweis einer Stellungnahme aus; mehr noch, es wird suggeriert, dass die Frage unzulässig sei. ${ }^{47}$ Nachgedoppelt wird mit dem Hinweis, dass das Material von 2005, das im Internet leicht aufzuspüren ist, nicht ,gerichtsfest“" sei. Ein Recht auf Vergessen sollte in diesem Fall, in dem es um Reputation und um die Zu- oder Aberkennung öffentlicher Ehre geht, meines Erachtens nicht zum Tragen kommen, weder juristisch, noch ethisch.

Das Ansinnen, in diesem Fall ein Recht auf Vergessen geltend zu machen, ist in vieler Hinsicht problematisch. Da es sich um eine Person des öffentlichen Interesses handelt, die das Recht beansprucht, würde auch aufgrund des EuGHUrteils vom Frühjahr 2014 dem Recht auf Vergessen vor Gericht kaum stattgegeben. Die bisherige Rechtsprechung innerhalb der Schweiz spiegelt jedenfalls diese Einschätzung wider: Das Recht, vergessen zu werden, kann nur dort sinnvoll zum Einsatz kommen, wo andernfalls eine Resozialisierung nachhaltig erschwert oder verunmöglicht ist. Es ist hingegen dort nicht $\mathrm{zu}$ verteidigen, wo es darum geht, durch Verschweigen und Verbergen von Informationen einen öffentlichen Reputationsverlust zu vermeiden. Die Urteile in den hier früher erwähnten Fällen von $1996^{48}$ respektive von $1985^{49}$ bestätigen diese Unterscheidung einerseits in Formen von Vergessen richtiger Informationen zugunsten von Resozialisierungsmöglichkeiten und andererseits in Formen von Vergessen korrekter Inhalte zugunsten des Erhalts von Ansehen und Ehre. Das Urteil des EuGH vom 13. Mai 2014 steht zu diesen Entscheiden in keinem Spannungsverhältnis, sondern bestätigt im Gegenteil diese juristische Praxis auch im Kontext des Digitalen.

46 Interview am 7.11.2014. http://www.srf.ch/news/schweiz/die-sp-nationalraetin-und-ihresteuertricks (Zugriff: 2.1.2016).

47 Im Mai 2006 berichtete die NZZ über die hier angesprochene Mietrechtsaffäre. Diese öffentliche Darstellung wurde damals von der Politikerin nicht gerichtlich angefochten. In der NZZ heißt es dazu: „Der Deutschschweizer Mieterinnen- und Mieterverband (MV) hat die SP-Nationalrätin Margret Kiener Nellen zum Rücktritt aus dem Vorstand aufgefordert. Sie sei nicht mehr tragbar gewesen, nachdem sie als Vermieterin selber gegen das Mietrecht verstossen habe, teilte der Verband am Donnerstag zu einem Bericht der ,Berner Zeitung، mit.“ http://www.nzz.ch/aktuell/ startseite/newzzEND1XP69-12-1.33088 (Zugriff: 2.1.2016).

48 Vgl. BGE 122 III 449.

49 Vgl. BGE 111 II 209. 
Aus einer ethischen Perspektive ist die Diskrepanz zwischen Formen von Vergessen zugunsten von Resozialisierungsbemühungen und solchen zugunsten der Durchsetzung von Zensur hervorzuheben. Von diesen beiden Formen des Vergessens zu unterscheiden sind zudem Forderungen nach Rehabilitierung. Diese umfasst nicht in erster Linie Vergessensvorgänge, sondern eine besonders intensive Erinnerungsleistung. Die Forderung nach sozialer Rehabilitierung würde in ihrem Kernanliegen verraten, wenn sie an ein Recht auf Vergessen gekoppelt oder als ein Recht auf Vergessen ausgelegt würde. Im Zusammenhang mit Rehabilitierung wäre im Gegenteil über ein Recht auf umfassendes Erinnern nachzudenken. Rehabilitierung ist die Wiederherstellung der Ehre einer Person aufgrund der Einbeziehung und/oder Neueinschätzung historischer Anhaltspunkte und Quellen. ${ }^{50}$ Wer sie für sich selbst oder für andere fordert, verlangt nach einer möglichst detaillierten, wahrheitsgetreuen Aufarbeitung historischer Sachverhalte und erwartet dadurch eine bisher verweigerte, aber einer Person womöglich zustehende, öffentliche Anerkennung. Die Wiederherstellung von Integrität und Ehre gelingt, indem bisher übersehene oder aber neue Hinweise in den Blick gerückt werden und sich so die Sicht auf dasselbe grundlegend ändert.

Zusammenfassend lässt sich noch einmal festhalten: Legitim kommt das Recht auf Vergessen zugunsten von Resozialisierungsbemühungen zum Einsatz, wenn damit Mitteilungen wieder in Informationen transformiert werden sollen. Nicht legitim geschieht der Rekurs auf ein Recht auf Vergessen hingegen, wenn Informationen als Informationen verdeckt gehalten werden und möglichst nie zu einer Mitteilung werden sollen, weil sonst die Reputation Schaden nähme. Legitim hingegen, und allerdings von der vorhergehenden Forderung klar zu unterscheiden, ist jene nach einem Recht auf Erinnern. In diesem Fall geht es um Fragen der Rehabilitierung. Die Forderung nach Rehabilitierung geht dahin, dass Informationen endlich zu Mitteilungen werden sollen, dass jemandem nachträglich öffentlich Recht getan und die Ordnung wieder hergestellt wird. Resozialisierung und Rehabilitierung beinhalten soziale Transformationsprozesse. Zensur und Ideologie hingegen beabsichtigen, solche Prozesse in beide Richtungen zu unterbinden.

50 Vgl. diesbezüglich das Bundesgesetz vom 20.6.2003 über die Aufhebung von Strafurteilen gegen Flüchtlingshelfer zur Zeit des Nationalsozialismus (SR 371) sowie das Bundesgesetz vom 20.3.2009 über die Rehabilitierung der Freiwilligen im Spanischen Bürgerkrieg (SR 321.1). Bei beiden geht es um die rückwirkende Aufhebung von rechtskräftigen und (in der Regel) längst vollzogenen Strafurteilen, die aus heutiger Sicht das Gerechtigkeitsempfinden in schwerwiegender Weise verletzen; vgl. dazu etwa Trechsel/Lieber (2013), vor Art. 381, Rz. 10. Bis Ende 2006 bestanden sodann im bürgerlichen wie auch im militärischen Strafrecht Verfahren zur Rehabilitation bei ausgesprochenen Nebenstrafen, die die Resozialisierung erleichtern sollten; vgl. Botschaft vom 21.9.1998 zur Änderung des Schweizerischen Strafgesetzbuches, BBl 1999 2210-2211. 
Gewisse Informationen dürfen nicht und unter keinen Umständen zu Mitteilungen werden, andere dürfen nicht und unter keinen Umständen aus dem Blick rücken.

Jemandem gesetzlich eine zweite Chance zuzugestehen, heißt auch, einen gesellschaftlichen Konsens darüber zu haben, dass in irgendeiner Hinsicht eine erste Chance nicht gelingend wahrgenommen worden ist oder werden konnte. In Kenntnis der Tatsache, dass eine Chance vertan ist, und nach bereits realisierten gesellschaftlichen Sanktionen ermöglicht das Recht auf Vergessen womöglich die Verwirklichung einer zweiten Chance, die Möglichkeit auf ein gelingendes Leben innerhalb der Gesellschaft. Wo ein Recht auf Vergessen dazu nötig wird, ist es nicht nur juristisch, sondern auch ethisch in Betracht zu ziehen.

\section{Literatur}

Abbt, C. (2016, i. E.), „Ich vergesse.“ Über Möglichkeiten und Grenzen des Denkens aus philosophischer Perspektive, Frankfurt am Main u. New York.

Aebersold, P. (2009), Ist das Ziel der Resozialisierung noch zeitgemäss?, in: Queloz, N., Luginbühl, U., Senn, A., Magri, S. (Hg.), Ist das Ziel der Resozialisierung noch zeitgemäss? Beiträge und Dokumentation der 6. Freiburger Strafvollzugstage (November 2008), Bern, 17-36, URL: http://www.avenirsocial.ch/sozialaktuell/sozial_aktuell_3977_3979.pdf (2.1.2016).

Boehme Nessler, V. (2008), Unscharfes Recht. Überlegungen zur Relativierung des Rechts in der digitalisierten Welt, Berlin.

Brägger, B. F. (2013), in: Niggli, M. A., u. Wiprächtiger H., (Hg.), Strafrecht I, Basler Kommentar, Basel 2013, 3. Aufl.

Cornel, H. (2009), Zum Begriff Resozialisierung, in: ders., Kawamura-Reindl, G., Maelicke, B., u. Sonnen, B. R. (Hg.), Resozialisierung, Baden-Baden, 3. Aufl.

Cornel, H. (2012), Recht der Resozialisierung. Strafrecht, Strafvollzug, Strafvollstreckung, Opferentschädigung, Sozialrecht, Baden-Baden.

Esposito, E. (2002), Soziales Vergessen. Formen und Medien des Gedächtnisses der Gesellschaft, übers. v. Corti, A., Frankfurt am Main.

Foucault, M. (1988), Un „fantastique“ de bibliothèque [1970], in: ders., Schriften zur Literatur, Frankfurt am Main, 157-177.

Frischknecht, J., Haffner, P., Haldimann, U., u. Niggli, P. (1984), Die unheimlichen Patrioten. Politische Reaktion in der Schweiz [1979], Zürich.

Guyer, P. (2009), Ist und Soll. Von Hume bis Kant, und heute, in: Klemme, H. F. (Hg.), Kant und die Zukunft der europäischen Aufklärung, Berlin u. New York, 210-231.

Häfliger, M. (2014), Wasser predigen und Wein trinken mit Steuerpolitikerin Kiener Nellen, Neue Zürcher Zeitung 6.11.2014, URL: http://www.nzz.ch/schweiz/wasser-predigen-undwein-trinken-mit-steuerpolitikerin-kiener-nellen-1.18419992 (2.1.2016).

Henning, C. (2015), Freiheit, Gleichheit, Entfaltung. Die politische Philosophie des Perfektionismus, Frankfurt am Main. 
Kutter, I. (2012), Das Netz vergisst doch - nur leider nicht das, was wir möchten, in: Die Zeit, 27.9.2012, URL: http://www.zeit.de/2012/40/Internet-Digitales-Gedaechtnis-DatenSpeicherung (2.1.2016).

Lindner, M. (2014), Privatheit im Informationszeitalter. Ethische Grundlagen von Privatheit und Anwendungsfragen in der Informationstechnologie, Tübingen, 58-115.

Maaßen, J. (2015), Metaphysik und Möglichkeitsbegriff bei Aristoteles und Nikolaus von Kues. Eine historisch-systematische Untersuchung, Berlin u. Boston.

McArthur, R. (2001), Reasonable expectations of privacy, in: Ethics and Information Technology 3, 123-128.

Mayer-Schönberger, V. (2010), Delete. Die Tugend des Vergessens in digitalen Zeiten, übers. v. Kamphuis, A., Berlin.

Nolte, N. (2011), Zum Recht auf Vergessen im Internet, in: Zeitschrift für Rechtspolitik 44, 236-240.

Oetker, H. (1994), Die Verjährung. Strukturen eines allgemeinen Rechtsinstituts, Baden-Baden.

Pethes, N. (2008), Kulturwissenschaftliche Gedächtnistheorien zur Einführung, Hamburg.

Ryan, J. (2013), History of the internet and the digital future, London.

Schaber, P. (2012), Menschenwürde, Stuttgart.

Schefczyk, M. (2012), Verantwortung für historisches Unrecht. Eine philosophische Untersuchung, Berlin u. New York.

Schweizer, R. J. (2014), in: Ehrenzeller, B., Schindler, B., Schweizer, R. J., u. Vallender, K. A. (Hg.), Die schweizerische Bundesverfassung, St. Galler Kommentar, Zürich u. St. Gallen, 3. Aufl.

Tavani, H. T. (2007), Philosophical theories of privacy. Implications for an adequate online privacy policy, in: Metaphilosophy 38, 1-22.

Trechsel, S., u. Lieber, V. (2013), in: Trechsel, S., u. Pieth, M. (Hg.), Schweizerisches Strafgesetzbuch. Praxiskommentar, Zürich u. St. Gallen.

Weber, R. H. (2011), The Right to Be Forgotten. More than a Pandora's Box, in: JIPITEC 2.2, 120-130, URL: http://www.jipitec.eu/issues/jipitec-2-2-2011/3084/jipitec\%202\%20-\%20 a\%20-\%20weber.pdf (2.1.2016).

Weber, R. H., u. Heinrich, U. I. (2014), Verletzt das Recht auf Vergessen(werden) des EuGH die Meinungsäusserungsfreiheit?, in: Jusletter IT 11.

Wöhe, B. (2015), Eigene Spuren aus dem Internet löschen - geht das?, URL: http://www. channelpartner.de/a/eigene-spuren-aus-dem-internet-loeschen-geht-das,2383909 (2.1.2016).

Zimmer, M. (2010), „But the data is already public“. On the Ethics of Research in Facebook, in: Ethics and Information Technology 12, 313-325. 